\title{
The Cascading Problem in a Water Distillation Plant for Heavy Water Production
}

\author{
By E. Cerrai, M. Silvestri and S. Villani \\ Laboratori CISE, Milano \\ (Z. Naturforschg. 11 a, 694-701 [1956]; eingegangen am 17. September 1955)
}

\begin{abstract}
A criterion for the designing of minimum specific volume cascades is generalized to be applicable in the case of large enrichments of the stages.

The practical solution of the cascading problem is also described for a water distillation plant for heavy water production which is supposed to use geothermal steam from the Larderello zone (Tuscany), taking into account the variability of the separation factor within the columns, due to the pressure drop.
\end{abstract}

It is well known that the water distillation process for heavy water production requires large amounts of steam. Therefore, apart from the enormous plant size, that process itself would be prohibitive if the heat for evaporation should be provided by means of fuel. The economics may be substantially improved if geothermal steam or low grade steam is available as a source of heat. The problem is then to check the minimum volume of the plant subordinately to several technical conditions. With well dimensioned cascades and highly efficient distillation towers the production cost could not be quite unreasonable.

As it happened already in other countries ${ }^{1}$, also in Italy we had in mind to use the geothermal steam in the Larderello (Tuscany) zone for the production of heavy water by means of distillation. The pressure of the steam coming out from the holes near Larderello is about $4-5$ abs. atm, and it is slightly superheated ${ }^{2}$. At present the steam is employed for power production (about $1.9 \cdot 10^{9} \mathrm{kWh}$ per year) and for the extraction of the boric acid, contained at a low concentration.

For the production of $50 \%$ dilute heavy water starting from the natural concentration it was suggested to use low pressure steam from exhausted holes (about 1.5 abs. atm; $110^{\circ} \mathrm{C}$ ) without interfering with power production. The steam supply was consequently limited.

For technical reasons the condensation pressure at the top of the columns was fixed at about 0.09 abs. atm $\left(44^{\circ} \mathrm{C}\right)$; in the range of the operation

1 Chem. Engng. News, Vol. 32, 3105 [1954].

2 A. Mazzoni, The steam vents of Tuscany and the Larderello plants, 2-nd edition, Calderini, Bologna, 1954. pressures the value of the separation factor is strongly affected by the pressure. For this reason we derived the enrichment ratio of a column taking into account the variability of the separation factor.

This work has been done considering ideal columns, i. e. constituted by theoretical plates, and it is easily extended to various kinds of columns, also of the packing type like the Spraypak towers ${ }^{3}$, provided that the H.E.T.P. and the pressure drop per plate are known.

\section{The separation factor}

Let us call separation factor for deuterium in the distillation process the ratio

$$
K=\frac{N /(1-N)}{n /(1-n)},
$$

where $N$ is the deuterium molar fraction in the water both in the form HDO and $\mathrm{D}_{2} \mathrm{O}$; and $n$ is the same in the vapour in equilibrium with the liquid phase. At low deuterium concentrations it is simply

$$
K=N / n \text {. }
$$

Since light water and heavy water solutions are ideal solutions, applying the Dalton law to the vapour phase and the RAOULT law to the equilibrium between the two phases, at low deuterium concentrations it follows that

$$
K=p_{\mathrm{H} 2 \mathrm{O}} / p_{\mathrm{HDO}},
$$

$p_{\mathrm{H} 2 \mathrm{O}}$ and $p_{\mathrm{HDO}}$ being the vapour pressures of the two kinds of water molecules.

3 Industrial Distillation development at Harwell, Atomics, Vol. 5, no. 8, p. 227 [1954]. 
At higher deuterium concentrations the expression (1) is not replaceable by the simpler one (2), but it can be shown that the separation factor is still expressed by the relationship (3) independently of the molar fraction, provided the following assumptions are made: a) the value of the equilibrium constant $K_{\mathrm{D}}$ of the exchange reaction

$$
\mathrm{H}_{2} \mathrm{O}+\mathrm{D}_{2} \mathrm{O} \rightleftharpoons 2 \mathrm{HDO} \text { is } K_{\mathrm{D}}=4,
$$

independently of temperature; $\mathrm{b})$ the vapour pressure of $\mathrm{HDO}$ is ${ }^{4} p_{\mathrm{HDO}}=\left(p_{\mathrm{H} 2 \mathrm{O}} \cdot p_{\mathrm{D} 2 \mathrm{O}}\right)^{1 / 2}$.

The separation factor is therefore depending on temperature only, according to the relationship (3), since the dependence on temperature $T$ is different for the vapour pressure of each molecular kind.

The empirical relationship, between $K$ and the absolute temperature $T$,

$$
K=0.866 e^{63.3 / T}
$$

holds satisfactorily in a temperature range of about 100 degrees above $283^{\circ} \mathrm{K}$.

Since the temperature $T$ and the pressure $p$ of saturated steam are univocally related, another empirical function, holding in the same range, can be written by means of the function $p=p(T)$. This empirical function will be

$$
K=0.866\left(\frac{7.705 \cdot 10^{8}}{p}\right)^{0.01266} .
$$

In Fig. 1 the values of $K$ and $p$ are plotted against the centigrade temperature.

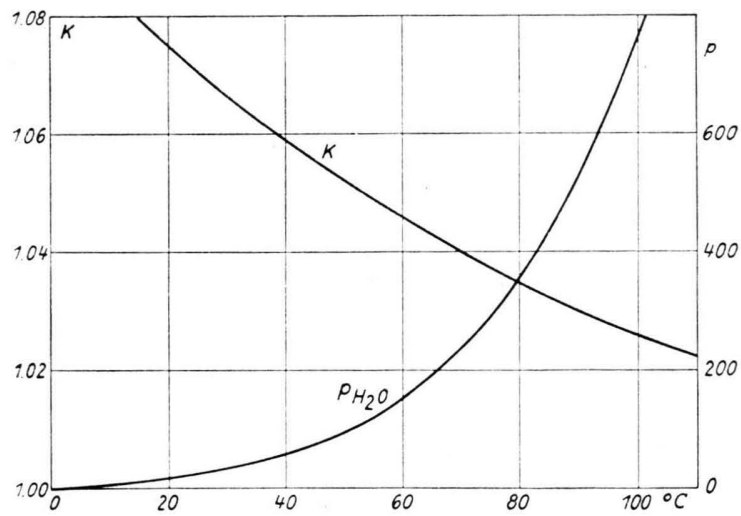

Fig. 1. Separation factor $K$ and pressure $p$ of saturated steam against the centigrade temperature.

\section{Fundamental parameters of the plant}

Our plant was assumed to consist of a certain number of stages, each of them constituted by distil-

${ }^{4}$ I. Kirshenbaum, Physical properties and analysis of heavy water, McGraw-Hill, New York, 1951, pp. 20-29. lation towers, which are composed by a given number of theoretical plates. A portion of the input of each stage is extracted enriched and feeds the subsequent stage, while the depleted vapour leaving the top of the stage is condensed and re-evaporated in the preceding one.

In Fig. 2 a sketch of the generical stage $i$-th is shown. The cross section of the column representing the stage could be the total section of a certain number of columns connected in parallel.

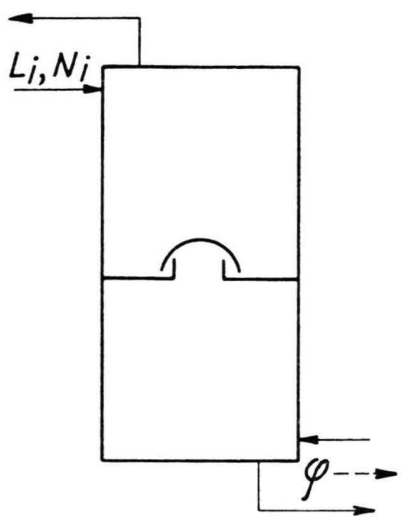

Fig. 2. Scheme for the generical $i$-th stage of the cascade.

Let us call $L_{i}$ the molar flow-rate of the water feeding the $i$-th stage, $N_{i}$ the corresponding deuterium molar fraction, and $a_{i}$ the enrichment ratio of the stage. $L_{p}$ and $N_{p}$ are respectively the molar flowrate and the molar fraction of the final product of the plant: then the quantity $\varphi=N_{p} L_{p}$ is the net transport of the isotope along the whole plant.

In the stages of the plant, far away from the output, the mass flow-rate of water is nearly the same as the mass flow-rate of vapour, the only difference being the quantity $L_{p}=\varphi / N_{p}$, that is very small compared with them. We call absolute yield of the stage the ratio

and

$$
\eta_{i}=\varphi / L_{i} N_{i}
$$

$$
\varrho_{i}=\eta_{i} K /(K-1)
$$

the relative yield referred to the yield of a column having an infinite number of plates.

\section{Cascading criteria}

We note first that the optimal cascading can be limited to that portion of the plant, which processes the material up to a deuterium concentration of a 
few parts percent. The remaining part of the plant for the final enrichment has a negligible size compared with the first portion.

We assume therefore to limit the optimal cascading in the range of deuterium molar fractions from $1.5 \cdot 10^{-4}$ to $4.5 \cdot 10^{-2}$ : the enrichment ratio $I$ of this portion of plant is then $I=300$.

Let us start dealing with the problem, taking into consideration a minimum volume plant criterion, which is suitable when the steam cost is relatively low compared with the capital charge. Such a criterion has been published by MARCHETTI ${ }^{5}$ in a case which is too restrictive for our purposes: it solves the cascading problem when the enrichment per stage is no more than 3 . Since the plant portion to be optimalized must provide an enrichment equal to 300 , if it were shared, for instance, into three stages, it is easily seen that the cited treatment would not be used.

It is therefore necessary to deal with the problem of the minimum volume plant criterion for any value of the enrichment. This philosophy, which is not the most general as the plant volume is considered to be constituted simply by the volume of the columns, leads nevertheless to useful indications about the choice of a convenient number of stages for the plant.

We assume at first the separation factor $K$ to be constant along the columns. According to Marchetri the specific volume of the stage (i. e. the volume per unit of product) is proportional to the quantity

$$
V_{i}=\frac{L_{i} S_{i}}{L_{i} \varrho_{i}}=\frac{1}{\varrho_{i}} \cdot \frac{\ln \left[\left(a_{i}-\varrho_{i}\right) /\left(1-\varrho_{i}\right)\right]}{\ln K} .
$$

The minimum value of $V_{i}$ with respect to $\varrho_{i}$ given by $\partial V_{i} / \partial \varrho_{i}=0$, is obtained when the following relationship between the enrichment and the yield holds:

$$
\frac{\varrho_{i}\left(a_{i}-1\right)}{\left(a_{i}-\varrho_{i}\right)\left(1-\varrho_{i}\right)}=\ln \frac{1-\varrho_{i}}{a_{i}-\varrho_{i}} .
$$

In Fig. 3 the values of $a_{i}$ and $\varrho_{i}$ satisfying the eq. (9) are plotted.

The specific volume of the portion of plant constituted by the first $m$ stages, which is to be optimalized, is proportional to the expression

$$
\left(\sum_{i=1}^{m} L_{i} S_{i}\right) / \varphi,
$$

5 C. Marchetti, Z. Naturforschg. 9 a, 1012 [1954].

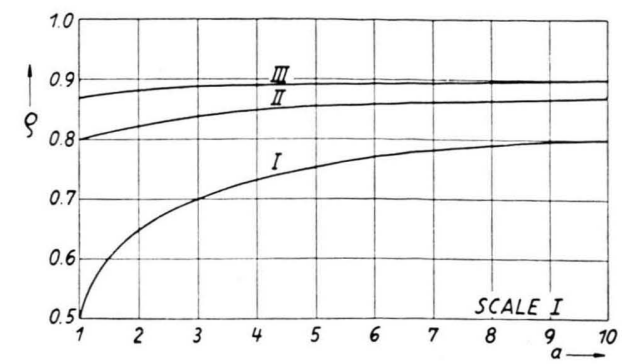

Fig. 3. Optimal relative yield of a stage as a function of the stage enrichment.

Scale II $=$ Scale I $\times 10 ;$ Scale III $=$ Scale I $\times 100$.

and therefore to the quantity

$$
V=\sum_{i=1}^{m} \frac{1}{N_{i} \varrho_{i}} \ln \frac{a_{i}-\varrho_{i}}{1-\varrho_{i}} .
$$

The optimal cascading of the portion composed of the first $m$ stages and producing a total enrichment $I$, is described by the following relationships:

$$
\left\{\begin{array}{l}
\frac{\partial V}{\partial N_{i}}=0 \quad(i=2,3, \ldots, m) \\
\prod_{i=1}^{m} a_{i}=I
\end{array}\right.
$$

We have

$$
V_{N_{i}}^{\prime}=\frac{\partial V}{\partial N_{i}}+V_{a_{i}}^{\prime} \frac{\partial a_{i}}{\partial N_{i}}+V_{a_{i-1}}^{\prime} \frac{\partial a_{i-1}}{\partial N_{i}}=0,
$$

where the symbol $V^{\prime}$ represents the substantial derivative of $V$ with respect to the subscript. It is therefore

$$
V_{a_{i}}^{\prime}=\frac{\partial V}{\partial a_{i}}+\frac{\partial V}{\partial \varrho_{i}} \frac{\mathrm{d} \varrho_{i}}{\mathrm{~d} a_{i}} .
$$

From eqs. (12), (13) it can be easily seen that the derivation involves only two terms of the function $V$. The calculation of the partial derivatives, recalling the condition $\partial V_{i} / \partial \varrho_{i}=0$ and the subsequent eq. (9), leads to

$$
\frac{1}{\varrho_{i}} \frac{a_{i}{ }^{2}}{a_{i}-\varrho_{i}}=\frac{1}{\varrho_{i+1}\left(1-\varrho_{i+1}\right)}, \quad(i=1,2, \ldots, m-1) .
$$

This expression relates the enrichment $a_{i}$ of the $i$-th stage to the enrichment $a_{i+1}$ of the succeeding stage, of which the quantity $\varrho_{i+1}$ is a function according to eq. (9).

When the enrichments $a_{i}$ of all the $m$ stages and the corresponding relative yields $\varrho_{i}$ are evaluated, the dimensions of that part of plant producing the total enrichment $I=a_{1} a_{2} \ldots a_{m}$ are determined. 
Practically, in order to calculate the optimal cascading according to the above shown theoretical development, for the last stage of the portion to be optimalized an arbitrary but reasonable value of the enrichment $a_{m}$ can be prefixed. By means of eq. (9) the value of $\varrho_{m}$ is obtained, and then through the relationship (14) the value of $a_{m-1}$ can be evaluated. In the same way all the values of the enrichments of the $m$ stages can be subsequently calculated. Generally, the product of such values will not give exactly $I$, because $a_{m}$ was chosen arbitrarily, but the right values can easily be reached through successive attempts.

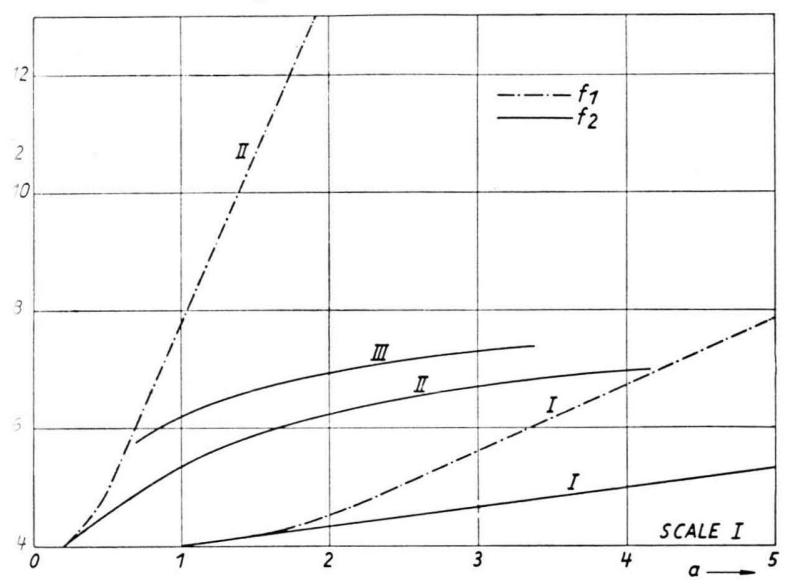

Fig. 4. Auxiliary functions for the best cascading calculations. - - - - $f_{1} ;-f_{2}$.

Scale II $=$ Scale I $\times 5 ;$ Scale III $=$ Scale $\mathrm{I} \times 10$.
To make easier the evaluation of the roots of eq. (14), two auxiliary functions have been tabulated. They are

$$
f_{1}\left(a_{i}\right)=\frac{1}{\varrho_{i}} \frac{a_{i}^{2}}{a_{i}-\varrho_{i}}
$$

and

$$
f_{2}\left(a_{i+1}\right)=\frac{1}{\varrho i+1(1-\varrho i+1)} .
$$

Their values are plotted in the graph of Fig. 4.

\section{Minimum volume cascades}

The enrichments per stage $a_{i}$ and the relative yields $\varrho_{i}$ have been calculated with the method above described, for cascades having all nearly the same enrichment $I=300$, but shared in $5,4,3,2$, 1 stages respectively.

The values of $V, a_{i}, \varrho_{i}$ and $N_{i}$ (starting from the natural deuterium concentration) for the various cascades are given in Table 1.

The data for $V$ clearly show that the specific plant volume decreases as the number of stages rises.

It is now interesting to calculate the quantity $V$ for the cascade composed of an infinite number of stages $(m \rightarrow \infty)$ and producing the same enrichment $I=300$. The value of $V$ for this cascade is obviously the lowest limit of the specific volume for

\begin{tabular}{|c|c|c|c|c|c|c|c|}
\hline \multicolumn{4}{|c|}{$V=27,970$} & \multicolumn{4}{|c|}{$V=28,700$} \\
\hline$i$ & $a_{i}$ & $N_{i}$ & $\varrho i$ & $i$ & $a_{i}$ & $N_{i}$ & $\varrho i$ \\
\hline $\begin{array}{l}1 \\
2 \\
3 \\
4 \\
5 \\
6\end{array}$ & $\begin{array}{l}1.85 \\
2.02 \\
2.40 \\
3.45 \\
9.7\end{array}$ & $\begin{array}{ll}1.5 & \cdot 10^{-4} \\
2.775 \cdot 10^{-4} \\
5.601 \cdot 10^{-4} \\
1.345 \cdot 10^{-3} \\
4.64 \cdot 10^{-3} \\
4.5 \cdot 10^{-2}\end{array}$ & $\begin{array}{l}0.635 \\
0.650 \\
0.675 \\
0.717 \\
0.798\end{array}$ & $\begin{array}{l}1 \\
2 \\
3 \\
4 \\
5\end{array}$ & $\begin{array}{r}2.06 \\
2.52 \\
3.9 \\
15.0\end{array}$ & $\begin{array}{l}1.5 \cdot 10^{-4} \\
3.09 \cdot 10^{-4} \\
7.79 \cdot 10^{-4} \\
3.036 \cdot 10^{-3} \\
4.49 \cdot 10^{-2}\end{array}$ & $\begin{array}{l}0.650 \\
0.680 \\
0.730 \\
0.815\end{array}$ \\
\hline \multicolumn{4}{|c|}{$m=3$} & \multicolumn{4}{|c|}{$m=2 \quad(I=300)$} \\
\hline$i$ & $a_{i}$ & $N_{i}$ & $\varrho i$ & $i$ & $a_{i}$ & $N_{i}$ & $\varrho i$ \\
\hline $\begin{array}{l}1 \\
2 \\
3 \\
4\end{array}$ & $\begin{array}{r}2.66 \\
4.5 \\
25.4\end{array}$ & $\begin{array}{l}1.5 \cdot 10^{-4} \\
3.99 \cdot 10^{-4} \\
1.795 \cdot 10^{-3} \\
4.56 \cdot 10^{-2}\end{array}$ & $\begin{array}{l}0.685 \\
0.740 \\
0.830\end{array}$ & $\begin{array}{l}1 \\
2 \\
3\end{array}$ & $\begin{array}{l}5.15 \\
58\end{array}$ & $\begin{array}{l}1.5 \cdot 10^{-4} \\
7.73 \cdot 10^{-4} \\
4.5 \cdot 10^{-2}\end{array}$ & $\begin{array}{l}0.755 \\
0.855\end{array}$ \\
\hline \multicolumn{4}{|c|}{$m=1 \quad V=59,300$} & \multicolumn{4}{|c|}{$\varrho=0.887$} \\
\hline
\end{tabular}
any possible plant.

Table 1. Characteristic quantities for some cascades with minimum specific volume. 
For the cascade shared into a very high number of stages all the enrichments $a_{i}$ are of course very close to unity, then we can write (infinitesimal case) : $a_{i}=1+\mathrm{d} a=1+\mathrm{d} N / N$, and from eq. (9) $\varrho=\frac{1}{2}(1+\mathrm{d} a / 2)$. Therefore the function $V$ defined by eq. (10) becomes:

$$
V=4 \int_{N_{1}}^{N_{p}} \frac{\mathrm{d} N}{N^{2}}=\frac{4}{N_{1}}\left(1-\frac{1}{I}\right) .
$$

Since in our case $I=300$, our limiting value is $V=26.578$.

According to the above described cascading criterion the height of the stages is progressively increasing. It can be interesting to compare the specific volumes of such cascades with that of cascades formed by stages of the same height. For this purpose we evaluated $V$ by means of eq. (10) also for the following two cases: 1) total enrichment $I=300$ produced by $m$ stages of the same height, each stage having an enrichment $a=\sqrt[m]{I}$ and a yield $\varrho$ related to $a$ by means of eq. $(9) ; 2$ ) the same conditions and feed are practically the same in each stage. Thus the heat consumption is given by the quantity

$$
\sum_{i=1}^{m} L_{i} / \varphi
$$

to which the expression

$$
C=\sum_{i=1}^{m} 1 / N_{i} \varrho_{i}
$$

is proportional through the factor $K /(K-1)$.

In Table 3 are shown the values of $C$ evaluated for each of the cascades considered above, according to the figures of Table 1 .

The specific steam consumption of a cascade formed by an infinite number of stages is infinite, because the depleted steam leaving any stage is evaporated an infinite number of times.

In the same Table 3 , the third column shows the values of quantity $C$ for plants having the same yields per stage as those listed in Table 1 , but without steam condensation between succeeding stages:

\begin{tabular}{|l|c|c|c|c|c|}
\hline$m$ & $V_{A}$ & $V_{B}$ & $\left\{\begin{array}{l}a_{B} \\
\varrho B\end{array}\right.$ & $V_{C}$ & $\left\{\begin{array}{l}a_{C} \\
\varrho C\end{array}\right.$ \\
\hline 1 & 59.300 & 59.300 & $\left\{\begin{array}{l}300 \\
0,887\end{array}\right.$ & 69.100 & $\left\{\begin{array}{l}300 \\
0,989\end{array}\right.$ \\
\hline 2 & 34.500 & 38.800 & $\left\{\begin{array}{c}17,33 \\
0,820\end{array}\right.$ & 43.180 & $\left\{\begin{array}{c}17,33 \\
0,958\end{array}\right.$ \\
3 & 30.260 & 32.920 & $\left\{\begin{array}{l}6,69 \\
0,775\end{array}\right.$ & 35.500 & $\left\{\begin{array}{l}6,69 \\
0,916\end{array}\right.$ \\
4 & 28.700 & 30.400 & $\left\{\begin{array}{l}4,16 \\
0,735\end{array}\right.$ & 32.900 & $\left\{\begin{array}{l}4,16 \\
0,878\end{array}\right.$ \\
5 & 27.970 & 29.150 & $\left\{\begin{array}{l}3,129 \\
0,706\end{array}\right.$ & 29.850 & $\left\{\begin{array}{l}3,129 \\
0,844\end{array}\right.$ \\
\hline
\end{tabular}

Table 2. Comparison between cascades composed by $m$ stages:
A) minimum specific volume: varying stage height;
B) unvaried stage height: $\varrho$ optimal;
C) unvaried stage height: $\varrho>\varrho$ optimal.

except for the yield $\varrho$, which has been chosen higher than the optimal value derived from eq. (9) in such a way to give a number of plates about $30 \%$ more than in case 1 . The data are shown in Table 2.

In order to get an idea of the steam consumption of the minimum specific volume cascades, we evaluate now the volume of water evaporated per volume unit of product. We recall that the vapour returned from a stage to the preceding one is condensed and evaporated again, and that for the first $m$ stages the mass flow rates of evaporated water

\begin{tabular}{|c|c|c|}
\hline$m$ & $\begin{array}{c}C \\
\text { with steam } \\
\text { condensation }\end{array}$ & $\begin{array}{c}C \\
\text { without steam } \\
\text { condensation }\end{array}$ \\
\hline 1 & 7,510 & 7,510 \\
2 & 10,321 & 8,534 \\
3 & 13,791 & 9,740 \\
4 & 17,128 & 10,260 \\
5 & 20,003 & 10,500 \\
$\infty$ & $\infty$ & 13,334 \\
\hline
\end{tabular}

Table 3. Figures for the quantity $C$ (proportional to the specific heat consumption) for some cascades with minimum specific volume. 
for the plant composed of an infinite number of stages the value of $C$ has been calculated assuming the limiting value $\varrho=1 / 2$.

The minimum value of the absolute specific consumption is ideally reached in a plant constituted by a single distillation tower infinitely high. This value is a useful figure to be compared with the consumption of any real plant. In the case of small isotope withdrawal, we see that the specific consumption has the expression $K /(K-1) N_{1}$, therefore the quantity $C$ has the value

$$
C=1 / N_{1}=6.667 \text {. }
$$

The data obtained above show that it is not convenient to exceed in the number of stages in the minimum specific volume cascades, because the diminishing of the plant size does not make up any longer for the increasing specific cost of the plant, due to the higher number of stages, and moreover for the increasing heat consumption.

Practical reasons too, as the ease of operation, lead us not to exceed in the number of stages. A good choice of the number of stages of the portion to be optimalized can be $m=3$.

Our considerations about the sharing of the cascade do not change substantially even using other cascading criteria.

\section{Design criterion in the case of limited steam supply}

Since in our case the steam supply was not too large we designed, for the low concentration portion of the plant $(I=300)$, a cascade in three stages under the condition of minimum heat consumption.

The amount of steam must be exploited as well as possible: for this purpose the highest difference in temperature between the bottom and the top of the columns is to be utilized in each stage, taking into account the temperature dependence of the separation factor, and a convenient temperature drop in the reboilers. These facts lead us to fix the same number of theoretical plates $S$ for all the columns. The enrichments and the yields of such a cascade satisfying the conditions of minimum specific heat consumption will now be calculated.

We note that, since the height of the stages is fixed, the minimum heat consumption criterion leads also to the minimum specific volume with regard to all the cascades having the same height of columns, shared into three stages.
Recalling that in these first three stages of the plant the mass flow rates of steam and water are practically the same, the quantity $C$ defined above is given by the expression:

$$
C=\frac{1}{N_{1}}\left[\frac{1}{\varrho_{1}}+\frac{1}{a_{1} \varrho_{2}}+\frac{1}{a_{1} a_{2} \varrho_{3}}\right] .
$$

Since

$$
\varrho_{i}=\frac{K^{S}-a_{i}}{K^{S}-1},
$$

and $a_{1} a_{2} a_{3}=I$, it follows

$$
C=\frac{K^{S}-1}{N_{1}}\left[\frac{1}{K^{S}-1}+\frac{1}{a_{1}\left(K^{S}-a_{2}\right)}+\frac{1}{a_{1} a_{2} K^{S}-1}\right] .
$$

By making the derivatives $\delta C / \delta a_{1}$ and $\delta C / \delta a_{2}$ equal to zero, the following expressions for $a_{1}$ and $a_{2}$ are obtained respectively:

$$
\begin{aligned}
& a_{1}=\frac{K^{S}\left(1+K^{S / 2}\right)+I K^{-S}}{1+K^{S / 2}+K^{S}}, \\
& a_{2}=\frac{K^{2 S}+I\left(1+K^{-S / 2}\right)}{K^{S}\left(1+K^{S / 2}\right)+I K^{-S}} .
\end{aligned}
$$

The corresponding relative yields can be obtained from eq. (20). The optimalized cascade is then individuated in its size-determining parameters.

\section{The column with variable separation factor}

In the preceding consideration we assumed the separation factor $K$ to be a constant throughout the column.

In our case, since the vapour leaving the top of the tower is condensed at low pressure, the temperature varies noticeably from the bottom to the top, because of the pressure drop through the column, and correspondingly a considerable variation of the separation factor $K$ occurs.

We wish therefore to calculate the enrichment produced by a column operating with a separation factor varying from plate to plate.

Let us assume that in a generical plate $s$ the isotope equilibrium between water and vapour is attained (ideal column), so that, at low deuterium concentrations, the molar fraction $n_{s-1}$ of the vapour leaving the plate $s$ and the molar fraction $N_{s}$ of the liquid leaving the same plate, are related in the following way

$$
N_{s}=K_{s-1} n_{s-1},
$$

where $K_{s-1}$ is the value of the separation factor $K$ at the pressure $p_{s-1}$ existing upon the plate $s$. 
The scheme of the column and that of the generical plate $s$ are shown in Fig. 5 .

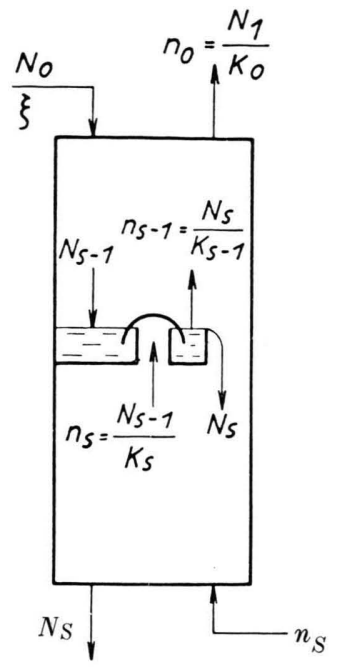

Fig. 5. Scheme for a column and its generic theoretical plate.

The eq. $n_{s}=N_{s-1} / K_{s}$ should be read $n_{s}=n_{s+1} / K_{s}$.

The plates are numbered from the top (plate 1) down to the bottom (plate $S$ ). The molar ratio of water to water vapour is called $\xi$. The isotope conservation relationship for the plate $s$ is given by

$$
\xi\left(N_{s}-N_{s-1}\right)=\frac{N_{s+1}}{K_{s}}-\frac{N_{s}}{K_{s-1}} .
$$

According to eq. (5), we write the separation factor $K_{s}$ in the form

$$
K_{s}=K_{0}\left(\frac{p_{0}}{p_{s}}\right)^{0.01266},
$$

where the subscript 0 refers to the top conditions.

Since the number of plates of the column is large, and the pressure drop in any plate is small com. pared with the overall pressure drop in the column, we can reasonably assume the variable $s$ to be continuous and the variability of $K$ with $s$ very small. Consequently in each couple of subsequent plates the separation factor can be considered to be unvaried $\left(K_{s}=K_{s-1}\right)$ and eq. (25) becomes more simply

$$
\xi\left(N_{s}-N_{s-1}\right)=\frac{1}{K_{s-1}}\left(N_{s+1}-N_{s}\right) .
$$

This approximation leads in many cases to a good accuracy in the corresponding integral formulae.

We put now

$$
\psi_{s}=N_{s+1}-N_{s} ;
$$

thus, eq. (27) can be written

$$
\xi K_{s-1} \psi_{s-1}=\psi_{s} .
$$

Since the separation effect of any plate is small, the dependence of the function $\psi_{s}$ on the variable $s$ is very slight; then one can write

$$
\psi_{s}=\psi_{s-1}+\psi_{s-1}^{\prime} \text {. }
$$

Hence the equation

$$
\frac{\psi^{\prime} s}{\psi_{s}}=\xi K_{s}-1
$$

generally holds and by integration we obtain:

$$
\psi_{s}=\psi_{0} \cdot \exp \left[\int_{0}^{s}\left(\xi K_{s}-1\right) \mathrm{d} s\right],
$$

where $\psi_{0}=N_{1}-N_{0}\left(N_{0}\right.$ being the feed concentration).

Let us put the concentration $N_{1}$ of the water in the first plate in the following form:

$$
N_{1}=N_{0}\left(1+\Theta \Delta K_{0}\right) \text {, }
$$

where $\Delta K_{0}=K_{0}-1$ and $\Theta(0 \leqq \Theta \leqq 1)$ is a parameter univocally related to the yield of the plant.

Since following the preceding assumptions we have

$$
\psi_{s}=\frac{N_{s+1}-N_{s}}{(s+1)-s}=\frac{\mathrm{d} N}{\mathrm{~d} s},
$$

eq. (32) becomes

$$
\frac{\mathrm{d} N}{\mathrm{~d} s}=N_{0} \Theta \Delta K_{0} \exp \left[\int_{0}^{s}\left(\xi K_{s}-1\right) \mathrm{d} s\right] .
$$

The integration leads to the following expression for the enrichment ratio $a=N_{S} / N_{0}$ of the column:

$$
\boldsymbol{a}=1+\Theta \Delta K_{0} \int_{0}^{S} \exp \left[\int_{0}^{s}\left(\xi K_{s}-1\right) \mathrm{d} s\right] \cdot \mathrm{d} s .
$$

The integrals in eq. (36) must be evaluated graphically, provided the function $K(s)$ is known.

It can be shown from the isotope balance in the whole plant that for values of $\xi$ very close to unity we may write

$$
\varrho=1-\Theta .
$$

The optimal cascading of a plant formed by columns in which $K$ is considered a constant can be extended to columns with variable $K$. This problem is solved by considering as equivalent two columns, the former having a value of $K$ decreasing from the 
top $\left(K_{0}\right)$ to the bottom $\left(K_{S}\right)$ and constituted by a number of plates $S$, the latter having a constant separation factor $K=K_{0}$ and a number of plates $\overline{S \text {, }}$ which produce the same enrichment $a$, and have the same relative yield $\varrho$ (and therefore the same absolute yield $\eta$ ).

The enrichment of the column with constant $K$ is given by

$$
a=\varrho+K_{0}^{\bar{S}}(1-\varrho)
$$

which is derived exactly and can be written, recalling eq. (37), in the form:

$$
a=1+\Theta\left[K_{0} \bar{s}-1\right] .
$$

As the enrichment of the two equivalent columns must be the same also in the case of no isotope withdrawal $(\varrho=0$, hence $\Theta=1$ and $\xi=1)$, from the comparison of eqs. (36) and (39) we obtain:

$$
K_{0} \bar{S}=1+\Delta K_{0} \int_{0}^{S} \exp \left[\int_{0}^{s} \Delta K_{s} \mathrm{~d} s\right] \cdot \mathrm{d} s .
$$

For a three stages plant the value of $K_{0} \bar{s}$ obtained in this way is to be substituted in eqs. (22) and (23) in order to evaluate the optimal enrichments of the stages with a given total enrichment $I$.

To give an idea of the difference between the number of plates evaluated assuming $K=$ const and that obtained from the method outlined (variable $K$ ) we make the following example. A column operates at a top pressure of $68.4 \mathrm{~mm} \mathrm{Hg}$, then with a top separation factor $K_{0}=1,0564$, and its type and performance are such as to give the function $K=K(s)$ plotted in Fig. 6. We fix an enrichment $a=21,5$ without isotope withdrawal $(\varrho=0)$. From eq. (36)

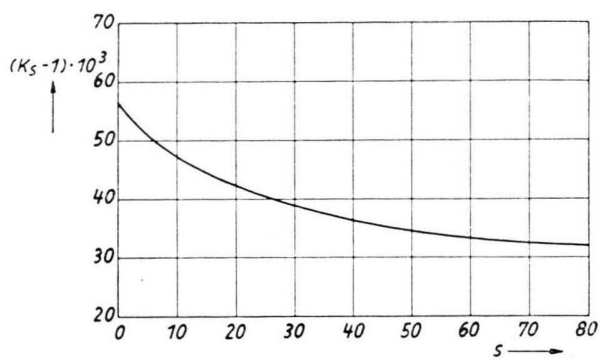

Fig. 6. The function $K(s)$ in a particular case.

we obtain $S=66,7$, while assuming a constant separation factor $\left(K=K_{0}\right)$ throughout the column we should obtain correspondingly, by means of eq. (38), the value $\bar{S}=56$. It can be noted that this comparison between $S$ and $\bar{S}$ holds for any value of the yield.

We could also derive the enrichment in the case of a constant separation factor $K=K_{0}$ from the approximate formula (36), which gives

$$
a=1+\Theta\left[e^{K_{0} \bar{S}}-1\right] .
$$

According to this formula an enrichment $a=21,5$ with a separation factor $K_{0}=1,0564$ and without isotope withdrawal would be reached by $S=54,2$ theoretical plates. 